\title{
Hierarchical Digital Control System Performance
}

\author{
Larkin Eugene VASILEVICH ${ }^{\mathrm{a}, 1}$, Akimenko Tatiana ALEKSEEVNA ${ }^{\mathrm{a}}$, Grishin \\ Konstantin ANATOLIEVICH ${ }^{\mathrm{a}}$ \\ ${ }^{a}$ Tula State University, Russia
}

\begin{abstract}
A hierarchical multiprocessor system designed to control complex production facilities is investigated. It is shown that distributed computing in such systems is performed at the strategic, tactical and functional-logical levels. Since the control process unfolds in real physical time, in which both digital controllers and a control computer operate, when developing software for such systems, the problem of estimating the time complexity of control algorithms arises. A matrix equation is obtained that describes a closed multi-loop control system, in which data asymmetry and pure delays are taken into account. It is shown that time delays worsen the characteristics of transient processes during the transition of an object to a steady state of operation. A method for soft estimation of time complexity is developed, based on the sequential simplification of the semiMarkov process, represented by the algorithm, with recalculation of its time characteristics at each step. It is shown that the method allows one to estimate both data distortions and delays in the feedback loops.
\end{abstract}

Keywords. Von Neumann controller, multiprocessing, hierarchical level, semiMarkov process, time interval, recursive procedure

\section{Introduction}

The complexity of the tasks performed implies the complexity of the structure of the technical means and the digital control system, which is expediently divided into hierarchical levels; strategic, tactical and functional-logical, each of which solves its own range of tasks [1-4]. At the top, strategic, hierarchical level, the tasks of deep production planning are solved, therefore, artificial intelligence or computer methods are used to make decisions at this level. In accordance with the plan, a sequence of tasks is formed, which are fed to the tactical level computer. A middle-level computer, having received a plan from a strategic computer, divides the general problem into tasks that go to the functional-logical level, where feedbacks are closed in fact through the actuators, the controlled object and sensors. If the computer division into strategic and tactical levels can be carried out virtually (depending on the complexity of the planning algorithm), then the division into tactical and functional-logical levels, as a rule, is hardware. This is determined by the properties of von Neumann controllers, which interpret control algorithms sequentially, operator by operator, which unfolds in real physical time [5]. Thus, the digital controller, in addition to the algorithmic

1 Corresponding Author, Larkin Eugene Vasilevich, Tula State University, Russia; E-mail: elarkin@mail.ru. 
implementation of the control law, contributes to delays in the control process, which, in turn, affects the quality characteristics of the control system as a whole [6 - 9]. In addition to the control loop, the scheduling problem only exacerbates the problem of time delays, degrades performance and can lead to a loss of system stability. To simulate time delays, one can use the apparatus of the semi-Markov process [10 - 14], since each control algorithm processes random data generated at the outputs of the sensors and includes decision-making operators at branch points, therefore, when interpreting the operator, the operation execution intervals are random.

\section{Configuration of the hierarchical multiprocessor control system}

Structure of hierarchical multiprocessor control system is shown on the figure. 1 [4].

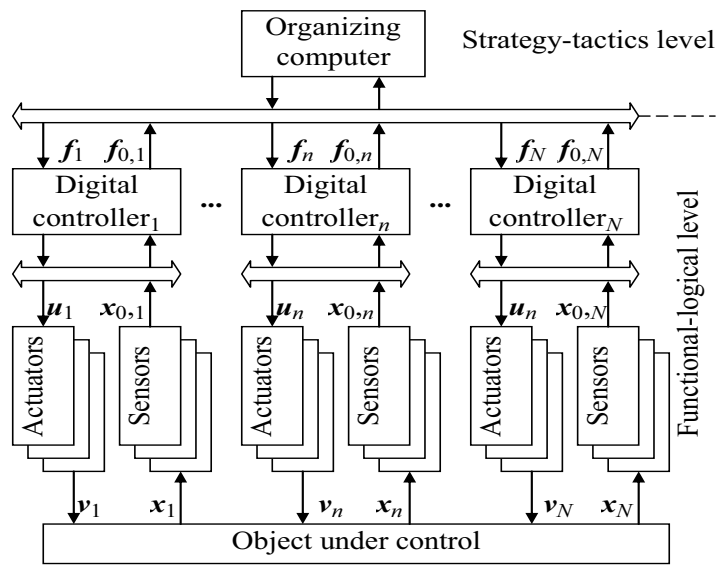

Figure. 1. Flowchart of hierarchical multiprocessor control system

On the flowchart strategy-tactics level is performed by organizing computer, whose destination is generating global aim of industry control system functioning, to dividing global problem onto local tasks and managing by controllers while solving assigned tasks. Every of $N$ digital controllers, forming the functional-logical level, is included into $k(n), 1 \leq n \leq N$ control loops, every of which, in turn, includes actuator, which provides proper action on a production installation, part of common technological process and sensor, which measures production process state. Object under control, actuated by control vectors $\mathbf{v}_{n}(t)=\left[v_{1(n)}(t), \ldots, v_{k(n)}(t), \ldots, v_{K(n)}(t)^{\theta}\right]$, where $\theta$ is matrix transpose sign, $t$ is the time, which, in turn, is transformed from vector $\mathbf{u}_{n}(t)=\left\lfloor u_{1(n)}(t), \ldots, u_{k(n)}(t), \ldots, u_{K(n)}(t)^{\theta}\right\rfloor$, calculated by the $n$-th controller, is characterized with state vector $\mathbf{x}_{n}(t)=\left[x_{1(n)}(t), \ldots, x_{k(n)}(t), \ldots, x_{K(n)}(t)\right]^{\theta}$, whose elements values are measured by sensors, which transform $\boldsymbol{x}_{n}(t)$ to feedback vector $\mathbf{x}_{0, n}(t)=\left[x_{0, n, 1(n)}(t), \ldots, x_{0, n, k(n)}(t), \ldots, x_{0, n, K(n)}(t)\right]^{\theta}$. The reference signal vector $\mathbf{f}_{n}(t)=\left[f_{1(n)}(t), \ldots, f_{k(n)}(t), \ldots, f_{K(n)}(t)\right]^{\theta}$ is transmitted to $n$-th controller from 
organizing computer. Organizing computer generates quests on the functional-logical level controllers by means of polling procedure, so algorithm, generated quests, is the cyclic one. Algorithms, realized in controllers, also are cyclic, they generate quests both to peripherals (sensors/actuators), and to organizing computer.

\section{Estimation of time intervals between transactions}

Performance of hierarchical multiprocessor control system highly depends of time intervals between transactions generated by the Von Neumann computer, for estimation of which model of polling procedure, unfolding in time, should be worked out [14 - 16]. Data in digital controller are processed according algorithms, which may be described as the $(N+1)$-parallel semi-Markov process

$$
\tilde{\mu}=\left\{\mu_{0}(t), \mu_{1}(t), \ldots, \mu_{n}(t), \ldots, \mu_{N}(t)\right\},
$$

where $\mu_{0}(t)$ describes organizing computer operation; $\mu_{n}(t)$ describes operation of $n$ th, $1 \leq n \leq N$, controller;

$$
\mu_{n}=\left\{A_{n}, \mathbf{h}_{n}(t)\right\}
$$

$A_{n}=\left\{a_{1(n)}, \ldots, a_{j(n)}, \ldots, a_{J(n)}\right\}$ are states of semi-Markov process $\mu_{n}(t)$, which simulate algorithm's operators; $\mathbf{h}_{n}(t)=\mathbf{g}_{n}(t) \otimes \mathbf{p}_{n}=\left\lfloor h_{j(n), l(n)}\right\rfloor$ is the $J(n) \times J(n)$ semiMarkov matrix; $\mathbf{g}_{n}(t)=\left\lfloor g_{j(n), l(n)}(t)\right\rfloor$ is the $J(n) \times J(n)$ matrix of pure time densities; $\mathbf{p}_{n}=\left\lfloor p_{j(n), l(n)}\right\rfloor$ is the $J(n) \times J(n)$ stochastic matrix.

With use methods, described in [15 - 17] on the semi-Markov process time delays between transactions may be obtained. Time delays are laid down at the stage of soft design, so, when simulation of the system it is necessary to forecast performance of the system, when at the digital controller such-and-such algorithm is realized with suchand-such time delays between transactions.

Let us consider $n$-th part of industrial object under managing of $n$-th digital controller. For simplification of analytical simulation it as more conveniently use instead description signal vectors $\mathbf{v}_{n}(t), \mathbf{u}_{n}(t), \mathbf{x}_{n}(t), \mathbf{x}_{0, n}(t), \mathbf{f}_{n}(t)$ their Laplace transforms are used, such as:

$$
\begin{aligned}
& \mathbf{V}_{n}(s)=L\left[\mathbf{v}_{n}(t)\right], \mathbf{U}_{n}(s)=L\left[\mathbf{u}_{n}(t)\right], \mathbf{X}_{n}(s)=L\left[\mathbf{x}_{n}(t)\right], \\
& X_{0, n}(s)=L\left[\mathbf{x}_{0, n}(t)\right], \mathbf{F}_{n}(s)=L\left[\mathbf{f}_{n}(t)\right],
\end{aligned}
$$

where $L[\ldots]$ is the direct Laplace transform operation, $s$ is the Laplace variable (differentiation operator) [18 - 20].

Input of vectors $\mathbf{F}_{n}(s)$ and $\mathbf{X}_{n, 0}(s)$ elements and output of vector $U_{n}(s)$ elements are executed in sequence, so in Von Neumann controller there are time intervals between transactions. Delays can be considered as abstract ones, without link with any time co-ordinate system. On the controller/object-under-control interface data are inputted/outputted with delays, nominated as follows:

$F_{n, k}(s)$ is inputted with the lag $\tau_{n, f, k}, 1 \leq k \leq K$;

$X_{n, 0, k}(s)$ is inputted with the lag $\tau_{n, 0, k}, 1 \leq k \leq K$; 
$U_{k}(s)$ is outputted with the lag $\tau_{n, u, k}, 1 \leq k \leq K$.

Such approach permits to express operation of the linear system through transfer function apparatus. Fully closed loops system is described with matrix equation $[2,3]$

$$
\mathbf{X}_{n}(s)=\mathbf{N}_{n, u}(s) \cdot \boldsymbol{\Phi}_{n}(s) \cdot\left[\mathbf{E}+\widetilde{\boldsymbol{\Phi}}_{n}(s)\right]^{-1} \cdot \mathbf{N}_{n, f}(s) \cdot \boldsymbol{\Phi}_{n, f, c}(s) \cdot \mathbf{F}_{n}(s),
$$

in which $\mathbf{N}_{n, u}(s)$ and $\mathbf{N}_{n, f}(s)$ are $K(n) \times K(n)$ diagonal matrices, describing data skews when transmitting data from digital controller to actuator and from reference signal vector to actuator; $\boldsymbol{\Phi}_{n}(s)$ is the matrix of transfer functions, describing $n$-th part of object under control; $\boldsymbol{E}$ is the $K(n) \times K(n)$ unit diagonal matrix; $\boldsymbol{\Phi}_{n, 0, c}(s)$ and $\boldsymbol{\Phi}_{n, f, c}(s)$ are $K(n) \times K(n)$ matrices of transfer functions, describing data processing by digital controller soft; $\widetilde{\boldsymbol{\Phi}}_{n}(s)$ is the $K(n) \times K(n)$ matrix of transfer functions, describing $n$-th part of object under control with data lags caused by sequential interpretation of control algorithm operators by Von Neumann type controller.

Matrices, describing delays in the system are as follows [18 - 20]:

$$
\begin{aligned}
& \mathbf{N}_{n, f}(s)=\left(\begin{array}{ccccl}
\exp \left(-\tau_{n, f, 1} s\right) & \ldots & \ldots & 0 \\
0 & \ldots & \exp \left(-\tau_{n . f, k} s\right) & \ldots & 0 \\
0 & \ldots & 0 & \ldots & \exp \left(-\tau_{n, f, K} s\right)
\end{array}\right) \text {; } \\
& \mathbf{N}_{n, 0}(s)=\left(\begin{array}{ccccl}
\exp \left(-\tau_{n, 0,1} s\right) & \ldots & 0 & \ldots & 0 \\
0 & \ldots & \exp \left(-\tau_{n, 0, k} s\right) & \ldots & 0 \\
& & \ldots & \ldots & \exp \left(-\tau_{n, 0, K} s\right) \\
0 & \ldots & 0 & 0
\end{array}\right) \\
& \mathbf{N}_{n, u}(s)=\left(\begin{array}{ccccl}
\exp \left(-\tau_{n, u, 1} s\right) & \ldots & 0 & \ldots & 0 \\
0 & \ldots & \exp \left(-\tau_{n, u, k} s\right) & \ldots & 0 \\
0 & \ldots & 0 & \ldots & \exp \left(-\tau_{n, u, K} s\right)
\end{array}\right) .
\end{aligned}
$$

where $\tau_{n . f, k}$ is the lag of appearance of $k$-th digital data $f_{k(n)}(t)$ relative to its continual counterparts; $\tau_{n, 0, k}$ is the lag of appearance of $k$-th digital data $x_{0, n, k(n)}(t)$ relative to its continual counterparts; $\tau_{n, u, k}$ is the lag of appearance of $k$-th analogue data $u_{k(n)}(t)$ relative to its digital counterparts.

Matrix $\widetilde{\boldsymbol{\Phi}}_{n}(s)$ describes object under control from interface controller/actuator till interface feedback/controller and is as follows:

$$
\widetilde{\boldsymbol{\Phi}}_{n}(s)=\mathbf{N}_{n, u}(s) \cdot \boldsymbol{\Phi}_{n, a}(s) \cdot \boldsymbol{\Phi}_{n}(s) \cdot \boldsymbol{\Phi}_{n, 0}(s) \cdot \mathbf{N}_{n, 0}(s),
$$


where $\quad \boldsymbol{\Phi}_{n, a}(s)=\left\lfloor\boldsymbol{\Phi}_{n, a, k(n), l(n)}(s)\right\rfloor \quad$ and $\quad \boldsymbol{\Phi}_{n, 0, k(n), l(n)}(s)=\left\lfloor\boldsymbol{\Phi}_{n, a, k(n), l(n)}(s)\right\rfloor$ are $K(n) \times K(n)$ diagonal matrices, describing actuators and sensors correspondingly, elements of which are as follows:

$$
\begin{aligned}
& \Phi_{n, a, k(n), l(n)}(s)=\left\{\begin{array}{l}
\Phi_{n, a, k(n)}(s), \text { when } k(n)=l(n) ; \\
0, \text { when } k(n) \neq l(n) .
\end{array}\right. \\
& \Phi_{n, 0, k(n), l(n)}(s)=\left\{\begin{array}{l}
\Phi_{n, 0, k(n)}(s), \text { when } k(n)=l(n) ; \\
0, \text { when } k(n) \neq l(n) .
\end{array}\right.
\end{aligned}
$$

As it follows from (4) complex exponent, characterizing delays are available both at the numerator, and at the denominator of closed loop transfer function. Those exponents, which are situated at numerator, born data skew at the control system, and insignificantly affect on the result of control on the functional-logical level, but may substantially affect on the performance of the Strategy-tactics level of the system. Those exponents, which are situated at denominator of closed loop transfer function directly influence at the quality characteristics of control, such as transient time, overshooting, and stability.

As for a simulation of strategy-tactics level, approach to modeling highly depends on the on the problems, solving by organizing computer. If it fully controls the operation of object through digital controllers, to simulation of it method, described above may be applied. If its function is the supervision of the process, then theory of relay-races may be used for it simulation [21].

\section{Example}

As an example, control of object with cross links, shown on the fig. 2, was considered. Feedback signals in the system are generated with inertia-less sensors, which measures state vector $\mathbf{X}(s)=\left[X_{1}(s), X_{2}(s)\right]^{\theta}$. In the system proportional unit feedback is realized, namely $\mathbf{U}(s)=\mathbf{F}(s)-\mathbf{X}(s)$.

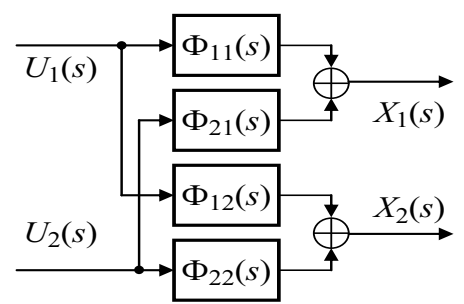

Figure. 2. Object under control

Transfer functions, defining object under control dynamics, are as follows:

$$
\Phi_{11}(s)=\Phi_{22}(s)=\frac{5}{0,02 s^{2}+0,3 s+1} ; \Phi_{12}(s)=\Phi_{21}(s)=\frac{1}{0,15 s+1} .
$$

System responses on standard control action $\mathbf{F}(s)=\left[L[1 \cdot 1(t), 0,5 \cdot 1(t),]^{\theta}\right.$, where $1(t)$ is the Heaviside function, are shown on the figure 3: 
Figure 3 a displays plots, when controller is the analogue one, and there are no delays at all, the closed loop system is stable, its overshooting does not exceed $15 \%$, the regulation time is less than $1 \mathrm{~s}$.;

Figure $3 \mathrm{~b}$ displays plots, when dispatching is carried out by organizing computer, delays of signals are equal to $0,01 \mathrm{~s}$, delays of signals are equal to $0,015 \mathrm{~s}$, data skew is absent. In this case overshooting increases till $25 \%$, regulation time increases till $2 \mathrm{~s}$, and it is necessary to complicate control algorithm to return to parameters, shown on the figure. 3 a.

Figure $3 \mathrm{c}$ displays plots, when delays are similar with delays on the figure. $3 \mathrm{~b}$, but at the sacrifice of data skew, being equal to $0,01 \mathrm{~s}$, on the plot of $\mathrm{x} 2$ drawdown emerging. That is additional destabilizing factor, which should be avoided with the control algorithm.

Figure $3 \mathrm{~d}$ displays plots, when control algorithm is realized on a single controller. In this case delays increase till 0,015 and $0,02 \mathrm{~s}$, respectively, and system is on the border of stability.
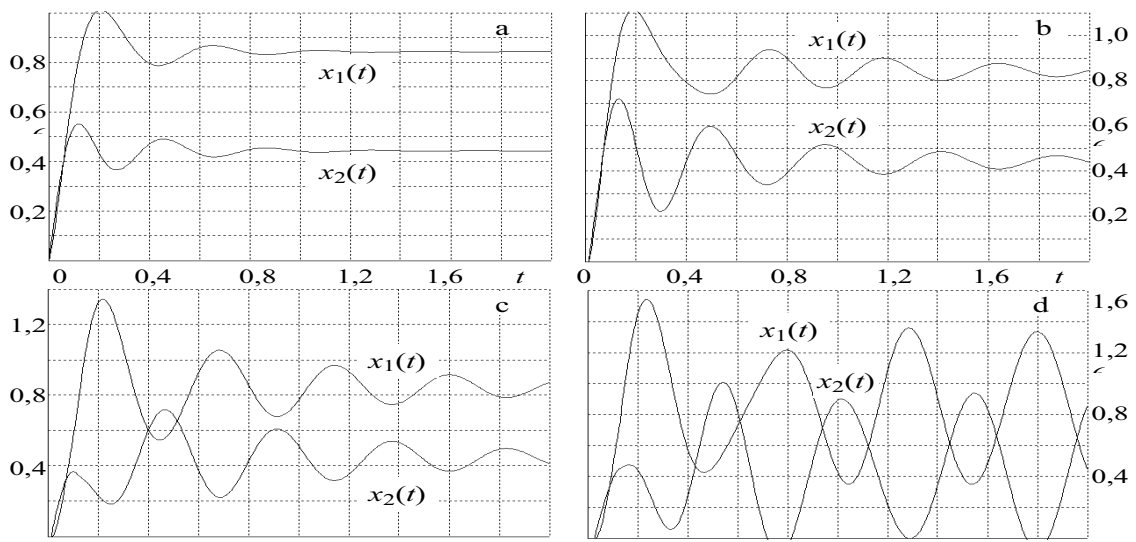

Figure. 3. Reaction of the system on the Heaviside function

\section{Conclusion}

As a result, a simple engineering method for estimating temporal characteristics is proposed. It is shown, that system hierarchical configuration permits to unload controllers from dispatching functions. It also reduces control loop latency and improves system performance. Using by designers the formula of closed loop system transfer function and the simplified method of time intervals estimation permit to forecast system properties on the configuration system working out stage. From this it follows that the time for preliminary debugging of the system will be reduced.

Further investigations in the domain may be directed to working out the model of interaction between organizing computer and controllers in time domain and methods. Second direction of investigation development is forming a genetic algorithm, which ensures guaranteed predetermined hierarchical digital control system performance. The reported study was funded by RFBR, project number 19-38-90066. 


\section{References}

[1] Malin Löfving M., Säfsten K.; Winroth M. Manufacturing strategy formulation, leadership style and organizational culture in small and medium-sized enterprises. IJMTM. 2016. Vol. 30. No. 5. Pp. 306 325.

[2] Landau I.D., Zito G. Digital Control Systems, Design, Identification and Implementation. Springer, 2006. 484 p.

[3] Aström J., Wittenmark B. Computer Controlled Systems: Theory and Design. Tsinghua University Press. Prentice Hall, 2002. 557 p..

[4] Kostenetskii P.S., Sokolinsky L.B. Simulation of Hierarchical Multiprocessor Database Systems // Programming and Computer Software/ 2013/ Vol. 39, No. 1, Pp. 10 - 24.

[5] Arnold K. A. Timing analysis in embedded systems // In Embedded hardware by J. Ganssler, K. Arnold et all. MA. 01803 USA. Elsevier Inc. 2008. Pp. 239 - 272.

[6] Zhang X.M., Min W.U., Yong H.E., Delay dependent robust control for linear systems with multiple time-varying delays and uncertainties // Control \& Decision, 2004. Vol. 19. N. 5. Pp. 496 - 500,

[7] Wu M., He Y., She J.H., Liu G.P. Delay-dependent criteria for robust stability of time-varying delay systems // Automatica, 2004. Vol. 40, N. 8. Pp. 1435 - 1439.

[8] Li D., Chen G. Impulses-induced p-exponential input-to-state stability for a class of stochastic delayed partial differential equations // International Journal of Control. 2019. Vol.: 92, N.: 8, Pp. 1805 - 1814.

[9] Sanz R., García P., Albertos P., Fridman E.Robust predictive extended state observer for a class of nonlinear systems with time-varying input delay // International Journal of Control. 2020. Vol. 93. N. 2 . Pp. 217 - 225.

[10] Bielecki T.R., Jakubowski J., Niewęgłowski M. Conditional Markov chains: Properties, construction and structured dependence // Stochastic Processes and their Applications. V. 127, N. 4. 2017. Pp. 11251170 .

[11] Ching W.K., Huang X., Ng M.K., Siu T.K. Markov Chains: Models, Algorithms and Applications / International Series in Operations Research \& Management Science. V. 189. Springer Science + Business Media NY, 2013. 241 p.

[12] Howard R. A. Dynamic Probabilistic Systems. Vol. 1: Markov Models. Vol. II: Semi-Markov and Decision Processes. Courier Corporation, 2012.

[13] Janssen J., Manca R. Applied Semi-Markov processes. Springer US, 2006. 310 p.

[14] Balsamo S., Harrison P.G., Marin A. Methodological construction of product-form stochastic Petri nets for performance evaluation // Journal of Systems and Software. Elsevier Inc. 2012. Vol. 85. № 7. Pp. $1520-1539$.

[15] Larkin E.V., Ivutin A.N. Estimation of Latency in Embedded Real-Time Systems // 3-rd Meditteranean Conference on Embedded Computing (MECO-2014). 2014. June 15-19. Budva, Montenegro, 2014. Pp. 236 - 239.

[16] Larkin E.V. Bogomolov A.V.; Privalov, A.N. A Method for Estimating the Time Intervals between Transactions in Speech-Compression Algorithms // Automatic Documentation and Mathematical Linguistics. 2017. Vol: 51. Iss. 5. Pp.: 214 - 219.

[17] Larkin, E., Ivutin, A., Esikov, D. Recursive Approach for Evaluation of Time Intervals between Transactions in Polling Procedure // 8th International Conference on Computer and Automation Engineering (ICCAE 2016). Australia, Melbourne. MATEC Web of Conferences, 56 (2016) 01004.

[18] Pavlov A.V. About the equality of the transform of Laplace to the transform of Fourier // Issues of Analysis. 2016. Vol.5(23). N.4(76). Pp. 21 - 30.

[19] Li J., Farquharson C.G., Hu X. Three effective inverse Laplace transform algorithms for computing time -domain electromagnetic responses // Geophysics. 2015. Vol. 81. N. 2. Pp. E75 - E90.

[20] Pospisisil M. Representation of solutions of delayed difference equations with linear parts given by pairwise permutable matrices via Z-transform // Applied mathematics and computation. 2017. V. 294. Pp. 180 - 194.

[21] Larkin, E.V., Privalov, A.N. The discrete analysis of relay-races. International Journal of Industrial and Systems Engineering, 2020, 35(1), Pp. 28-56 